\title{
Effect of type 2 diabetes mellitus on exercise intolerance and the physiological responses to exercise in peripheral arterial disease
}

\author{
S. Green • C. D. Askew • P. J. Walker
}

Received: 17 August 2006 / Accepted: 23 November 2006 / Published online: 20 January 2007

(C) Springer-Verlag 2007

\begin{abstract}
Aims/hypothesis There are conflicting data about the effect of type 2 diabetes mellitus on exercise tolerance in peripheral arterial disease. To elucidate this problem, we compared the tolerance and physiological responses to treadmill and cycle exercise in 31 patients with peripheral arterial disease and intermittent claudication.

Materials and methods One group of these patients had type 2 diabetes $(n=12)$ and its members were matched for sex and age with a group of patients who did not have diabetes $(n=12)$. Since BMI and body weight were greater in the diabetic group $\left(28.4 \pm 3.7\right.$ vs $25.2 \pm 2.4 \mathrm{~kg} / \mathrm{m}^{2} ; 84.0 \pm$ 14.6 vs $73.8 \pm 8.0 \mathrm{~kg}$ ), we also studied a third, 'heavy' group of non-diabetic patients with claudication of similar age $\left(n=7\right.$; BMI $=30.9 \pm 5.3 \mathrm{~kg} / \mathrm{m}^{2}$; body weight $=85.2 \pm$ $8.2 \mathrm{~kg})$.

Results Compared with the 'light' non-diabetic group, maximum treadmill times were shorter for the diabetic
\end{abstract}

S. Green $(\bowtie)$

School of Biological, Biomedical and Molecular Sciences,

University of New England,

Armidale, New South Wales, Australia

e-mail: simon.green@une.edu.au

C. D. Askew

Faculty of Health, Science and Education,

University of the Sunshine Coast,

Maroochydore, Queensland, Australia

P. J. Walker

Department of Surgery, The University of Queensland,

Brisbane, Queensland, Australia

S. Green

Department of Physiology, School of Medical Sciences,

University of Otago,

Dunedin, New Zealand and heavy non-diabetic groups (1,448 vs 845 and $915 \mathrm{~s}$; ANOVA $p=0.01$ ); maximum cycle time also tended to be shorter (ANOVA, $p=0.08$ ) in the diabetic and heavy nondiabetic groups $($ median $=1,231$ vs 730 and $797 \mathrm{~s}$ ). The majority of physiological responses assessed were not different between the groups, although the time constant of oxygen uptake during submaximal treadmill and cycle exercise was significantly larger (ANOVA $p<0.05$ ) for the diabetic group.

Conclusions/interpretation These data demonstrate that exercise tolerance is lower in diabetic than non-diabetic patients with claudication, but that this difference is due to obesity rather than diabetes itself.

Keywords BMI · Claudication · Cycling · Diabetes . Intolerance · Oxygen uptake · Peripheral arterial disease · Walking

$\begin{array}{ll}\text { Abbreviations } \\ \text { ABI } & \text { ankle/brachial index } \\ \mathrm{PAD} & \text { peripheral arterial disease } \\ \mathrm{RBWH} & \text { Royal Brisbane and Women's Hospital } \\ V \mathrm{CO}_{2} & \text { rate of carbon dioxide production } \\ V \mathrm{O}_{2} & \text { rate of oxygen uptake }\end{array}$

\section{Introduction}

Exercise intolerance is a major manifestation of atherosclerotic peripheral arterial disease (PAD), particularly when PAD results in intermittent claudication [1-4]. Intermittent claudication is ischaemic muscle pain evoked by exercise and relieved with rest; such exercise-limiting pain is often experienced in the calf muscles during walking and in the 
calf and/or quadriceps muscles during cycling [5]. The primary pathological change leading to intermittent claudication is the atherosclerotic narrowing of one or more of the major arterial vessels feeding the lower limbs, i.e. aorta, iliac, femoral, popliteal and/or tibial arteries, resulting in a reduction of the maximum blood flow to the lower extremities during exercise [6]. In addition, microcirculatory, neuromuscular and metabolic changes occur within the skeletal muscles of the lower extremities, and these could potentially contribute to exercise intolerance in patients with claudication [7]. Since the medical management of PAD should focus on improving exercise tolerance [8], it is imperative that we better understand the physiological causes of exercise intolerance in this patient group.

Type 2 diabetes mellitus is a major risk factor for PAD and intermittent claudication [9-11]. Approximately 5\% of individuals who develop asymptomatic or symptomatic PAD also present with diabetes [12-14]. Diabetes is relatively more prevalent in individuals with symptomatic PAD (i.e. intermittent claudication). For example, in the Framingham Heart Study, which followed individuals over a 38-year period, diabetes was more prevalent in subjects who developed intermittent claudication $(20 \%)$ than in individuals who did not develop claudication (6\%) [11].

Exercise tolerance and cardiorespiratory fitness are impaired in individuals with type 2 diabetes mellitus who do not have PAD or other clinical evidence of cardiovascular disease [15-17]. However, results on the effect of type 2 diabetes mellitus on exercise tolerance in individuals with PAD and intermittent claudication are conflicting. Pain-free and maximal times spent on a treadmill test were found to be lower in diabetic than in non-diabetic patients with claudication in one study [18], but were found to be similar in another [19]. Lower-extremity function, assessed using walking, strength and balance tests, was impaired in PAD patients with diabetes compared with those who did not have diabetes [20]. In this latter study, the poorer lowerextremity function in diabetic patients was associated with a higher incidence of neuropathy and cardiovascular disease.

In the study by Dolan et al. [20], diabetic PAD patients had a significantly lower frequency of exertional leg symptoms and intermittent claudication than non-diabetic PAD patients. An important question that remains unanswered is whether exercise tolerance is affected by type 2 diabetes mellitus in individuals who have PAD with intermittent claudication and whose exercise performance is not limited by symptoms other than claudication, as could have been the case in these other studies [18-20]. Moreover, there are only very limited data about the symptomatic and physiological responses to exercise that might help explain any differences in exercise tolerance between diabetic and non-diabetic patients with claudication [19]. Therefore, the aim of the present study was to compare exercise tolerance and physiological responses to exercise in diabetic and non-diabetic patients with claudication.

\section{Materials and methods}

Subjects All subjects gave their written informed consent to the experimental procedures, which were approved by the ethics committees of the Royal Brisbane and Women's Hospital (RBWH) and University of Queensland, Australia. Patients were recruited from the vascular outpatients' clinic at the RBWH. Thirty-one individuals with PAD (defined as having a resting ankle/brachial index $[\mathrm{ABI}]<0.9)$ and a history of stable intermittent claudication $(>1$ year) were studied. These patients were selected from a slightly larger sample of patients $(n=42)$ involved in an exercise training study that was described recently [21]. Of these patients with claudication, 12 had been diagnosed with type 2 diabetes mellitus, as confirmed by abnormally high blood glucose and glycosylated haemoglobin levels; these comprised the diabetic group in this study. These patients with claudication were matched for sex and were of a similar age to 12 other patients with claudication but without diabetes (non-diabetic group). Since BMI and body weight were different between the non-diabetic and diabetic groups (Table 1), a third group of patients with claudication was selected. This group did not have diabetes, but did have similar (i.e. increased) BMI and body weight to the diabetic patients. This third 'heavy' group of non-diabetic patients with claudication (heavy non-diabetic group) was chosen to shed light on the potential influence of body weight on any difference in exercise tolerance between the well-matched diabetic and non-diabetic groups. Unfortunately, the heavy non-diabetic group was smaller $(n=7)$ and had a different sex distribution (four women, three men) from the other two groups.

Patients were excluded from participation in the study if they presented with one or more of the following: unstable or exertional angina, resting limb pain, random syncope, orthopaedic injury, recent or impending surgery, uncontrolled hypertension, a coronary or cerebrovascular event within 1 year of the study, exercise ECG abnormalities, chest pain, dizziness. Physical characteristics and cardiovascular risk factors for these three groups of patients with claudication are shown in Table 1. Fasting venous blood measurements, including indices of type 2 diabetes mellitus (i.e. plasma glucose and glycosylated haemoglobin levels), are shown in Table 2.

Exercise testing All subjects completed a screening and familiarisation routine prior to the collection of data reported in this study. After performing an initial screening 
Table 1 Physical characteristics, cardiovascular risk factors and prescribed drug usage in the three groups of patients with claudication
${ }^{*} p<0.05$ for difference between non-diabetic and the other groups

\begin{tabular}{llll}
\hline & Diabetic & Non-diabetic & Heavy non-diabetic \\
\hline Men/women & $10 / 2$ & $10 / 2$ & $3 / 4$ \\
Age (years) & $63.6 \pm 6.7$ & $62.8 \pm 7.9$ & $57.7 \pm 14.9$ \\
Weight $(\mathrm{kg})$ & $84.0 \pm 14.6$ & $73.8 \pm 8.0^{*}$ & $85.2 \pm 8.2$ \\
BMI $\left(\mathrm{kg} / \mathrm{m}^{2}\right)$ & $28.4 \pm 3.7$ & $25.2 \pm 2.4^{*}$ & $30.9 \pm 5.3$ \\
Blood pressure $(\mathrm{mmHg})$ & & & \\
Systolic & $139.0 \pm 16.7$ & $134.4 \pm 18.2$ & $134.1 \pm 7.0$ \\
Diastolic blood & $71.8 \pm 6.9$ & $71.7 \pm 8.7$ & $71.9 \pm 8.5$ \\
ABI & & & \\
Best leg & $1.00 \pm 0.19$ & $1.01 \pm 0.25$ & $1.03 \pm 0.14$ \\
Worst leg & $0.67 \pm 0.11$ & $0.74 \pm 0.15$ & $0.77 \pm 0.14$ \\
Stroke $(n)$ & 4 & 1 & 0 \\
Myocardial infarction $(n)$ & 4 & 2 & 1 \\
Hypertension $(n)$ & 10 & 5 & 5 \\
Hyperlipidaemia $(n)$ & 5 & 10 & 3 \\
Current smoker $(n)$ & 2 & 5 & 3 \\
Past smoker $(n)$ & 10 & 6 & 3 \\
Medication $(n)$ & & & \\
Antihypertensives & 9 & 4 & 3 \\
Insulin & 4 & 0 & 0 \\
Oral hypoglycaemics & 7 & 0 & 0 \\
Anticoagulants & 8 & 10 & 4 \\
Statins & 5 & 10 & 3 \\
Beta blockers & 3 & 2 & 0 \\
Anti-anginals & 0 & & 1 \\
Asthma medications & & & \\
\hline & 5 & & \\
\hline
\end{tabular}

treadmill test to maximum claudication, subjects returned to the laboratory for a session aimed at familiarising them with all the testing apparatus and exercise protocols, including learning how to walk on the treadmill without handrail support. After these two sessions, they completed at least four maximal graded tests, two on a motorised treadmill and two on an electrically braked cycle ergometer, over a 2-week period. A third test on a given mode was conducted if there was more than $25 \%$ difference in maximum walking or cycling times between the two tests [22]. Performance on, and physiological responses to, the last treadmill and cycle test are reported in this study. Each testing session was preceded by a brief $(2 \mathrm{~h})$ period of fasting, during which the subjects were only allowed to drink water.

The maximal graded walking test was performed on a motorised treadmill (TrackMaster TMX425CP; Full Vision, Newton, KS, USA) at a constant speed of $2.7 \mathrm{~km} / \mathrm{h}$. The treadmill gradient was initially set at $0 \%$ for the first $5 \mathrm{~min}$ of the test, and then increased by $2 \%$ every 3 min until the patient failed to sustain the task. Pain-free walking time and the maximum time spent walking were recorded. This protocol is similar to that used previously by our group [1$3,5,23]$ and is highly reproducible (average $\mathrm{CV}=6 \%$ ) when conducted after the above-mentioned familiarisation routine [2]. The maximal graded cycle test was performed
Table 2 Fasting blood results for the three groups of patients with claudication

Units for variables are $\mathrm{mmol} / \mathrm{l}$ unless stated otherwise. $* p<0.05$ for difference between diabetic and the other groups

\begin{tabular}{lccc}
\hline & Diabetic & Non-diabetic & Heavy non-diabetic \\
\hline Glucose & & $4.8 \pm 0.5$ & $5.0 \pm 0.4$ \\
HbA $_{1 \mathrm{c}}(\%)$ & $9.7 \pm 3.9^{*}$ & $5.5 \pm 0.5$ & $5.2 \pm 0.4$ \\
C-reactive protein & $8.2 \pm 2.5^{*}$ & $5.8 \pm 2.8$ & $4.0 \pm 1.1$ \\
Homocysteine & $4.5 \pm 3.3$ & $12.1 \pm 4.5$ & $11.0 \pm 3.9$ \\
Cholesterol & $12.9 \pm 4.3$ & $4.4 \pm 0.7$ & $5.2 \pm 0.8$ \\
Triacylglycerol & $4.9 \pm 0.7$ & $1.6 \pm 1.1$ & $1.6 \pm 0.8$ \\
HDL & $3.5 \pm 2.0 *$ & $1.2 \pm 0.3$ & $1.4 \pm 0.2$ \\
Total cholesterol/HDL-cholesterol ratio & $1.2 \pm 0.2$ & $3.6 \pm 0.6$ & $3.8 \pm 0.7$ \\
LDL & $4.3 \pm 0.9$ & $2.5 \pm 0.6$ & $3.1 \pm 0.6$ \\
VLDL & $2.5 \pm 0.8$ & $0.7 \pm 0.5$ & $0.7 \pm 0.3$ \\
White blood cell count & $1.2 \pm 0.3 *$ & $7.4 \pm 1.3$ & $7.6 \pm 1.7$ \\
Packed cell volume (\%) & $9.2 \pm 5.7$ & $44 \pm 3$ & $45 \pm 4$ \\
\hline
\end{tabular}


on an electrically braked cycle ergometer (Lode Rehcor, Groningen, The Netherlands) at a cadence of $60 \mathrm{rpm}$. For the first 5 min of the test the power output was set at $30 \mathrm{~W}$, and thereafter it was increased $10 \mathrm{~W}$ each 3 min until the subject failed to sustain the required cadence. Pain-free cycling time and the maximum time spent cycling were recorded. During the treadmill and cycle tests, the site(s) and severity of claudication pain were assessed every $60 \mathrm{~s}$ and at the end of exercise using a pain severity scale $(0=$ no pain, 5 = maximum pain).

Physiological measurements Heart rate and pulmonary gas exchange data were collected for 2 min prior to exercise, while the subject was seated on the cycle ergometer or standing on the treadmill, as well as throughout exercise. Heart rate was measured with a portable heart rate monitor (S610i; Polar Electro-Oy, Kempele, Finland) and averaged over 5-s intervals. Minute ventilation, rates of oxygen consumption $\left(V \mathrm{O}_{2}\right)$ and carbon dioxide production $\left(V \mathrm{CO}_{2}\right)$, and the respiratory exchange ratio $\left(V \mathrm{CO}_{2} / \mathrm{VO}_{2}\right)$ were measured breath-by-breath and averaged over 5-s intervals (CPX/D; MedGraphics, St Paul, MN, USA). Submaximal gas exchange and heart rate values were calculated by averaging all 5-s samples recorded between the 3-min 50-s and 4-min 45-s time points of the first 5-min exercise stage. Peak values for these variables were the highest 15 -s averaged values recorded during the last $3 \mathrm{~min}$ of the exercise test.

The breath-by-breath $V \mathrm{O}_{2}$ responses during the first exercise stage were fitted with an exponential function, $V \mathrm{O}_{2}(t)=V \mathrm{O}_{2}(a)+b\left(1-e^{[-t-T D / \tau]}\right)$, where $V \mathrm{O}_{2}(t)$ is $V \mathrm{O}_{2}$ at time $t, V \mathrm{O}_{2}(a)$ is the resting baseline $V \mathrm{O}_{2}, b$ is the amplitude of the $V \mathrm{O}_{2}$ response from rest to steady state, TD is the time delay from the onset of exercise to the initiation of phase II, and $\tau$ is the time constant. The time constant $(\tau)$ represents the speed with which $V \mathrm{O}_{2}$ responds to exercise: a low time constant reflects a fast response; whereas a high time constant reflects a slow response. We excluded the first $40 \mathrm{~s}$ of data from analysis because this period, on average, corresponded to the duration of the first phase of the $V \mathrm{O}_{2}$ response in another group of claudicants we had studied [2], and this is a phase that does not accurately reflect metabolism in working skeletal muscle. Curve fitting and parameter estimation were made using weighted leastsquares non-linear regression techniques and TableCurve 2D software (1996; Jandel Scientific, San Rafael, CA, USA). Because of relatively noisy data and poor fitting, one subject from the non-diabetic group and two subjects from the diabetic group were excluded from the analysis of $\mathrm{VO}_{2}$ kinetics.

The ABI was measured in triplicate in both legs at the end of a 20-min period of rest. The ABI was calculated using the systolic pressures of the highest brachial artery and the higher of the dorsalis pedis or posterior tibial arteries in each leg, all of which were measured within $60 \mathrm{~s}$ of each other. An average resting ABI value for each leg was obtained by averaging the closest two of the triplicate measures.

Statistical analysis Comparisons of variables between groups were made using a one-way ANOVA when the data were normally distributed or a Dunn's non-parametric test when the data were not normally distributed. Values are reported as means and standard deviations, unless otherwise stated. Relationships between variables were established using Pearson's correlation coefficient. Statistical significance was set at $p \leq 0.05$. Statistical tests were not performed on the incidence data in Table 1 because the observed frequencies were too low.

\section{Results}

Subject characteristics The data in Tables 1 and 2 show that the three groups of patients were well matched for age, blood pressure and resting ABIs. The diabetic and heavy non-diabetic patients with claudication were significantly heavier and had a significantly higher BMI than the nondiabetic patients with claudication. The diabetic group had significantly higher fasting blood glucose, $\mathrm{HbA}_{1 \mathrm{c}}$, triacylglycerol and VLDL levels than the other groups. Only three
Table 3 The incidence of symptoms limiting performance during treadmill and cycle tests in the three groups of patients with claudication

\begin{tabular}{lccccccc}
\hline & \multicolumn{2}{l}{ Treadmill } & & & Cycle & \\
\cline { 2 - 4 } \cline { 6 - 8 } & Diabetic & $\begin{array}{l}\text { Non- } \\
\text { diabetic }\end{array}$ & $\begin{array}{l}\text { Heavy non- } \\
\text { diabetic }\end{array}$ & & Diabetic & $\begin{array}{l}\text { Non- } \\
\text { diabetic }\end{array}$ & $\begin{array}{l}\text { Heavy non- } \\
\text { diabetic }\end{array}$ \\
\hline Calf pain & 11 & 9 & 5 & & 3 & 7 & 3 \\
Quadricep pain & 2 & 2 & 0 & & 9 & 5 & 5 \\
Hamstring pain & 3 & 1 & 2 & & 0 & 1 & 0 \\
Buttock pain & 6 & 2 & 1 & & 0 & 0 & 1 \\
Dyspnoea & 1 & 3 & 0 & & 1 & 4 & 1 \\
General fatigue & 1 & 1 & 1 & & 1 & 0 & 1 \\
\hline
\end{tabular}


of the 12 diabetic subjects had been diagnosed with neuropathy by their physicians.

Exercise responses All subjects reported claudication pain during the treadmill and cycle tests. The number of symptoms that limited performance and were cited as the reasons for stopping exercise varied between one and three (shown in Table 3).

Maximum exercise times in the three groups were not normally distributed. A box plot of maximum exercise times on the treadmill and cycle tests is shown in Fig. 1. Maximum walking time was significantly shorter in the diabetic $($ median $=845 \mathrm{~s})$ and heavy non-diabetic $($ median $=$ $915 \mathrm{~s}$ ) groups than in the non-diabetic group (median $=$ $1,448 \mathrm{~s}$ ). There was also a tendency (ANOVA $p=0.08$ ) for maximum cycle time to be shorter in diabetic (median = $730 \mathrm{~s}$ ) and heavy non-diabetic (median $=797 \mathrm{~s}$ ) than in non-diabetic subjects (median $=1231 \mathrm{~s})$. Pain-free exercise times were not significantly different between diabetic, non-diabetic and heavy non-diabetic subjects on the treadmill (median $=255$ vs 278 vs 360 s) or cycle test ( 383 vs 510 vs 323 s). For all 31 subjects, BMI, but not body weight, was significantly correlated with maximum walking time (Fig. 2a). The correlation between BMI and maximum cycling time $(r=-0.22)$ was not significant.

Physiological responses prior to and during the exercise tests are shown in Table 4. Although the $V \mathrm{O}_{2}(\mathrm{l} / \mathrm{min})$ at rest and during submaximal exercise tended to be higher in diabetic and heavy non-diabetic than in non-diabetic subjects, these differences never achieved significance. Peak $V \mathrm{O}_{2}\left(\mathrm{ml} \mathrm{kg}^{-1} \mathrm{~min}^{-1}\right)$ during the treadmill and cycle tests tended to be lower in the diabetic and heavy non-

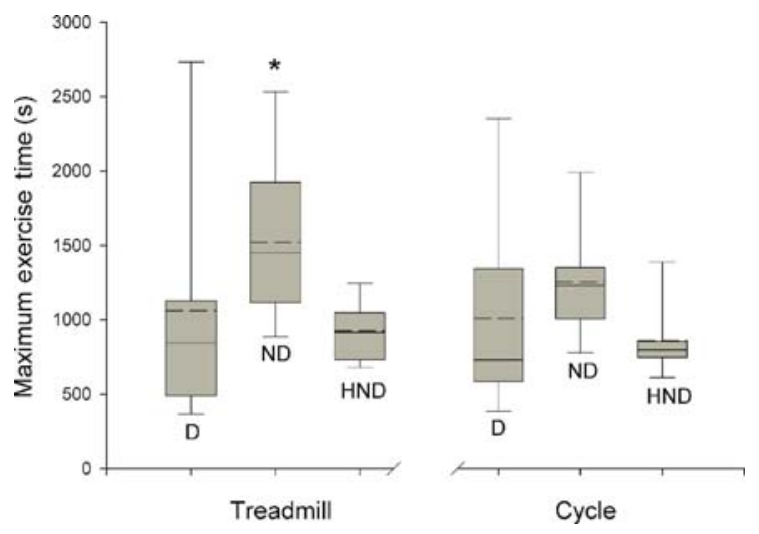

Fig. 1 Box plots of exercise performance times of the three groups of subjects with claudication: diabetic $(D)$, non-diabetic $(N D)$ and heavy non-diabetic $(H N D)$. The continuous horizontal line is the median. The upper or lower boundary of the box closest to the median represents the 25 th percentile, and the other boundary represents the 75 th percentile. Whiskers above and below the box represent the 90th and 10 th percentiles. The dashed horizontal line is the mean. ${ }^{*} p \leq 0.05$ for difference between the non-diabetic group and the other groups. $p=0.08$ (ANOVA) for between-group comparison (cycle)
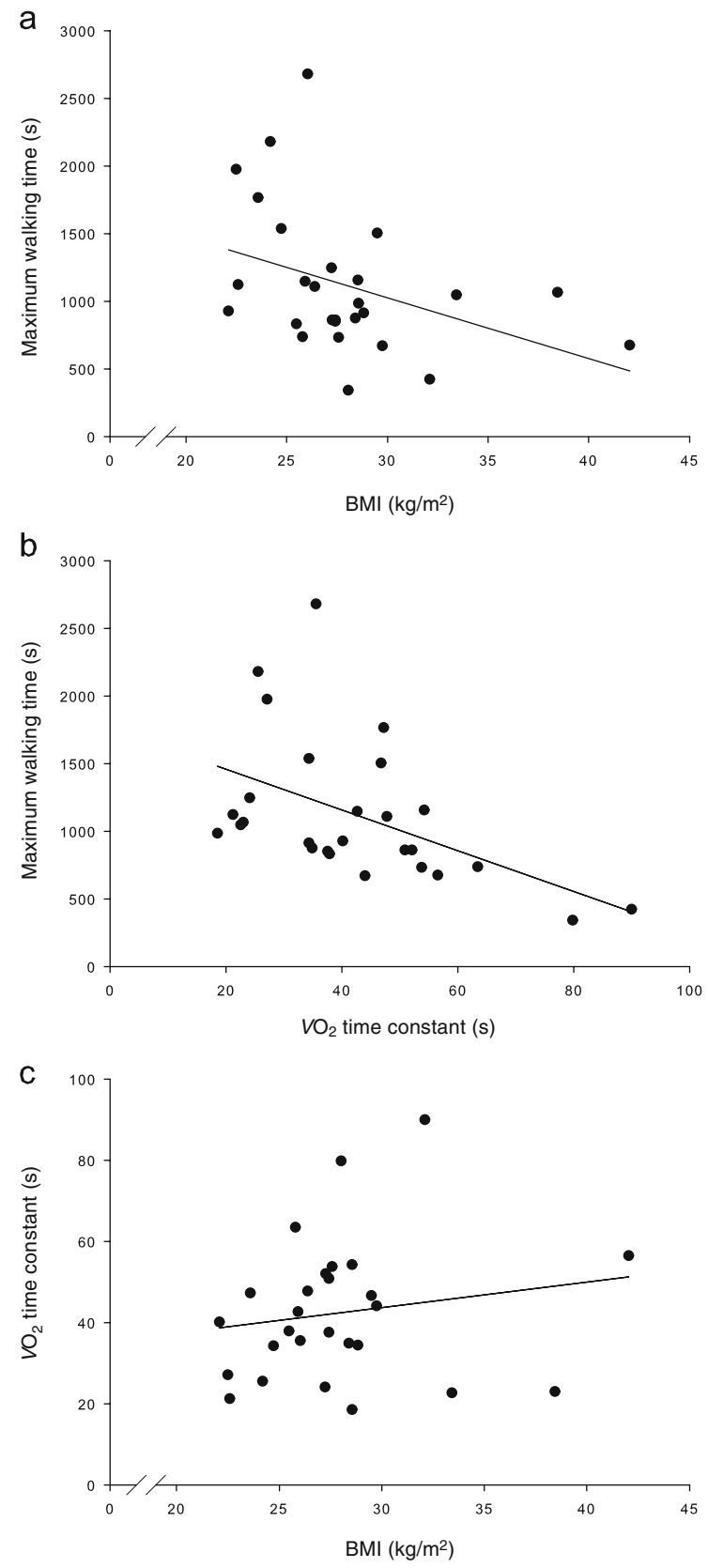

Fig. 2 a-c Scatterplots and correlations between maximum walking time, $\mathrm{BMI}$ and the time constant of $\mathrm{VO}_{2}$ during treadmill exercise in 28 patients with claudication from the three groups. $r=-0.38, p<0.05$ (a); $r=-0.49, p<0.05$ (b); $r=0.16, p>0.05$ (c)

diabetic than in the non-diabetic group, although these differences were not significant (ANOVA $p=0.12-0.20$ ). Parameters describing the kinetic response of $V \mathrm{O}_{2}$ during the first stage of the exercise tests are shown in Table 5. Baseline $V_{\mathrm{O}_{2}}$ (parameter a) for treadmill exercise was significantly larger in diabetic and heavy non-diabetic than in non-diabetic subjects, and the $V \mathrm{O}_{2}$ time constant was significantly larger in diabetic than in the other two groups. The $V \mathrm{O}_{2}$ time constant for submaximal cycling was also significantly larger in diabetic than in the other two groups. 
Table 4 Physiological responses prior to and during graded treadmill and cycle tests in the three groups of patients with claudication

\begin{tabular}{|c|c|c|c|c|c|c|}
\hline & \multicolumn{3}{|l|}{ Treadmill } & \multicolumn{3}{|l|}{ Cycle } \\
\hline & Rest & Submaximal & Maximal & Rest & Submaximal & Maximal \\
\hline \multicolumn{7}{|l|}{ Heart rate $(\mathrm{bpm})$} \\
\hline Diabetic & $80 \pm 16$ & $95 \pm 10$ & $121 \pm 27$ & $79 \pm 13$ & $96 \pm 11$ & $121 \pm 26$ \\
\hline Non-diabetic & $75 \pm 13$ & $88 \pm 12$ & $123 \pm 19$ & $75 \pm 14$ & $91 \pm 14$ & $129 \pm 24$ \\
\hline Heavy non-diabetic & $79 \pm 12$ & $92 \pm 12$ & $115 \pm 17$ & $75 \pm 13$ & $94 \pm 14$ & $115 \pm 16$ \\
\hline \multicolumn{7}{|l|}{$V \mathrm{O}_{2}(\mathrm{ml} / \mathrm{min})$} \\
\hline Diabetic & $317 \pm 77$ & $805 \pm 174$ & $1324 \pm 482$ & $313 \pm 71$ & $747 \pm 140$ & $1307 \pm 499$ \\
\hline Non-diabetic & $281 \pm 61$ & $721 \pm 140$ & $1303 \pm 290$ & $267 \pm 36$ & $679 \pm 63$ & $1274 \pm 273$ \\
\hline Heavy non-diabetic & $296 \pm 112$ & $772 \pm 116$ & $1152 \pm 158$ & $295 \pm 85$ & $694 \pm 65$ & $1065 \pm 186$ \\
\hline \multicolumn{7}{|l|}{$V \mathrm{O}_{2}\left(\mathrm{ml} \mathrm{kg}^{-1} \mathrm{~min}^{-1}\right)$} \\
\hline Diabetic & $3.8 \pm 0.6$ & $9.7 \pm 1.1$ & $15.9 \pm 5.7$ & $3.7 \pm 0.4$ & $9.0 \pm 1.0$ & $15.7 \pm 5.6$ \\
\hline Non-diabetic & $3.7 \pm 0.7$ & $9.5 \pm 1.4$ & $17.5 \pm 4.5$ & $3.6 \pm 0.5$ & $9.1 \pm 1.0$ & $17.1 \pm 4.1$ \\
\hline Heavy non-diabetic & $3.5 \pm 1.4$ & $9.1 \pm 1.7$ & $13.5 \pm 1.5$ & $3.5 \pm 1.0$ & $8.2 \pm 0.7$ & $12.6 \pm 2.0$ \\
\hline \multicolumn{7}{|l|}{ RER } \\
\hline Diabetic & $0.97 \pm 0.08$ & $0.95 \pm 0.08$ & $1.18 \pm 0.10$ & $0.97 \pm 0.06$ & $1.05 \pm 0.10$ & $1.28 \pm 0.13$ \\
\hline Non-diabetic & $0.92 \pm 0.05$ & $0.95 \pm 0.08$ & $1.20 \pm 0.10$ & $0.98 \pm 0.08$ & $1.04 \pm 0.09$ & $1.32 \pm 0.11$ \\
\hline Heavy non-diabetic & $0.89 \pm 0.10$ & $0.92 \pm 0.07$ & $1.14 \pm 0.07$ & $0.95 \pm 0.08$ & $1.02 \pm 0.09$ & $1.29 \pm 0.10$ \\
\hline \multicolumn{7}{|l|}{$\mathrm{VE}(1 / \mathrm{min})$} \\
\hline Diabetic & $12.1 \pm 3.0$ & $25.9 \pm 6.0$ & $51.3 \pm 19.3$ & $11.4 \pm 2.0$ & $23.2 \pm 8.1$ & $58.6 \pm 27.0$ \\
\hline Non-diabetic & $10.5 \pm 2.6$ & $23.2 \pm 6.2$ & $49.0 \pm 12.2$ & $10.2 \pm 1.8$ & $22.6 \pm 2.2$ & $55.2 \pm 8.5$ \\
\hline Heavy non-diabetic & $10.7 \pm 5.0$ & $22.7 \pm 5.7$ & $40.3 \pm 10.8$ & $10.5 \pm 3.6$ & $22.6 \pm 5.3$ & $43.0 \pm 10.3$ \\
\hline
\end{tabular}

There were no significant differences between groups. See Results for further details.

$R E R$ Respiratory exchange ratio; $V E$ minute ventilation

When all subjects were analysed, the $\mathrm{VO}_{2}$ time constant during treadmill exercise was significantly correlated with maximum walking time (Fig. 2b).

\section{Discussion}

Exercise tolerance is often measured as the maximum time sustained on a graded treadmill test. Previous studies have shown that exercise tolerance is severely impaired in patients with claudication and that, on average, maximum exercise times are $\sim 40$ to $50 \%$ of those values observed in healthy controls [1-4]. These data are based on studies that have excluded individuals with type 2 diabetes, yet a significant number of patients with claudication have type 2 diabetes (see Introduction). Very few studies have examined the effect of type 2 diabetes on exercise tolerance in patients with claudication, and findings from these few studies are conflicting [18-20].

In the current study, exercise tolerance was assessed in more detail than in these previous studies; we also used highly reproducible graded treadmill and cycle tests $[2,5]$. Subjects were selected from a cohort of 42 patients with claudication who participated in a recent exercise training study [21]. Twelve of these patients with claudication were in the diabetic group and they were matched for sex and age to 12 non-diabetic patients with claudication from the non-diabetic group.

On average, exercise tolerance was $40 \%$ lower in the diabetic than in the non-diabetic group, when it was assessed using either the graded treadmill or cycle test

Table 5 Parameters describing the kinetic response of $\mathrm{VO}_{2}$ during the first stage of the exercise tests

\begin{tabular}{|c|c|c|c|c|c|c|}
\hline & \multicolumn{2}{|c|}{ Diabetic $(n=10)$} & \multicolumn{2}{|c|}{ Non-diabetic $(n=11)$} & \multicolumn{2}{|c|}{ Heavy non-diabetic $(n=7)$} \\
\hline & Treadmill & Cycle & Treadmill & Cycle & Treadmill & Cycle \\
\hline Baseline $V \mathrm{O}_{2}(\mathrm{ml} / \mathrm{min})$ & $359 \pm 121$ & $312 \pm 61$ & $296 \pm 75^{*}$ & $259 \pm 32$ & $348 \pm 92$ & $272 \pm 24$ \\
\hline Amplitude (ml/min) & $533 \pm 169$ & $466 \pm 64$ & $441 \pm 76$ & $414 \pm 31$ & $480 \pm 79$ & $429 \pm 26$ \\
\hline Time constant (s) & $74 \pm 72 * *$ & $71 \pm 52 * *$ & $39 \pm 11$ & $37 \pm 11$ & $35 \pm 16$ & $38 \pm 16$ \\
\hline
\end{tabular}

${ }^{*} p<0.05$ for difference between non-diabetic and the other groups

$* * p<0.05$ for difference between diabetic and the other groups 
(Fig. 1). These findings substantiate other evidence of greater exercise dysfunction in PAD when diabetes is present $[18,20]$. However, body weight and BMI were significantly higher in the diabetic group, an effect also reported by others [16, 20]. Since an increase in body weight lowers exercise tolerance in older subjects [23], we included a second non-diabetic group in the present study, whose members were as heavy as the diabetic group. The fact that exercise tolerance in the heavy non-diabetic group was similar to that of the diabetic group, but lower than that of their lighter non-diabetic counterparts, suggests that the lower exercise tolerance in diabetic patients with claudication is more likely to be caused by higher body weight than by diabetes itself.

This interpretation is further supported by the significant, inverse correlation between exercise tolerance and BMI (Fig 2a). Although peak $V \mathrm{O}_{2}$ was not different between the groups, a heavier body weight tended to both increase the $\mathrm{O}_{2}$ cost of submaximal exercise and reduce peak $V \mathrm{O}_{2}$ relative to body weight $\left(\mathrm{ml} \mathrm{kg}^{-1} \min ^{-1}\right)$ (Tables 4 and 5). Both of these factors increase the relative intensity of exercise at a given workload and reduce the time that can be sustained on a graded test [23].

The kinetic response of $V \mathrm{O}_{2}$ during submaximal exercise (i.e. $\tau=$ time constant) is impaired in type 2 diabetes mellitus [16] and PAD $[2,24]$. The time constant of $\mathrm{O}_{2}$ uptake reflects the speed with which $V \mathrm{O}_{2}$ increases towards its steady rate, with a relatively higher value reflecting a relatively slow response. Our previous observations in PAD patients suggests that a slowing of this $\mathrm{VO}_{2}$ response during submaximal exercise might lower the tolerance of maximal graded exercise $[2,23]$. In the present study, the kinetic response of $\mathrm{VO}_{2}$ was impaired in the diabetic patients with claudication compared with the non-diabetic groups (Table 5). Moreover, there was a significant, inverse correlation between the $\mathrm{VO}_{2}$ time constant during treadmill walking and maximum walking time (Fig. 2b), as we had observed previously in a different group of patients with claudication [2]. This raises the possibility that the lower exercise tolerance in the diabetic group could be partly mediated by relatively slowed $V \mathrm{O}_{2}$ kinetics, an effect that is independent of the influence of BMI on exercise tolerance given the lack of association between BMI and the $V \mathrm{O}_{2}$ time constant (Fig. 2c). However, this possibility is weakened by our finding that exercise tolerance was similar between the diabetic and heavy non-diabetic groups, despite their different $\mathrm{VO}_{2}$ kinetics.

The severity of PAD (i.e. resting ABI) was not different between the groups and so it alone cannot explain the poorer exercise tolerance in the diabetic group. However, the ABI is a poor predictor of the lower-limb haemodynamic response to exercise $[25,26]$, and there is evidence of more severe atherosclerosis in distal segments of the lower extremity in diabetic compared with non-diabetic patients with claudication [27]. Therefore, we cannot exclude the possibility that muscle blood flow during exercise was more impaired in diabetic patients with claudication. The greater incidence of previous hypertension in the diabetic group (Table 1) compared with the well-matched non-diabetic group suggests that muscle phenotypic characteristics associated with hypertension, such as capillary rarefaction and a greater percentage of type II fibres, might also be present in diabetic skeletal muscle and further reduce exercise tolerance [1]. Since we did not formally test for neuropathy, we also cannot exclude the possibility that the more severe intolerance to exercise in the diabetic group is partly attributable to neuropathy.

Individuals with PAD or diabetes often have other diseases that lower exercise tolerance, such as coronary artery disease, heart failure, respiratory disease and concomitant orthopaedic/arthritic disabilities. In the study by Dolan et al. [20], the poorer functional status of diabetic PAD patients was attributed partly to a higher incidence of neuropathy and concomitant cardiovascular disease. The diabetic PAD patients in that study reported a lower frequency of exertional leg symptoms and intermittent claudication than non-diabetic PAD patients, and a higher frequency of resting leg pain. The diabetic patients in the present study were clearly different from those studied by Dolan et al. [20] because they all had intermittent claudication and did not report resting leg pain. Patients in the present study were only included if exercise tolerance was limited by claudication (Table 3 ); thus the exclusion criteria applied in the present study ensured that exercise tolerance was not limited by the above-mentioned coexisting diseases or other contraindications to exercise. Although there was a slightly higher incidence of previous stroke and myocardial infarctions in the diabetic group (Table 1), which might have lowered exercise tolerance, the average performance response for those diabetic patients who had experienced these clinical events was similar to the average for the entire diabetic group. A higher usage of antihypertensive and hypoglycaemic medication was observed in the diabetic group, but to our knowledge their effects on intermittent claudication and exercise tolerance are not known.

A limitation of the present study was the relatively small number of subjects $(n=31)$ tested, and so the findings should be considered as preliminary. Another limitation was the fact that the group of heavy non-diabetic patients with claudication had a relatively higher proportion of women than the other two groups. The 31 subjects tested in the present study were selected from a slightly larger group of 42 patients with claudication involved in an exercise training study [21], 18 of whom were women. Tolerance of graded treadmill exercise was not significantly different 
between these 18 women (median=923 s) and the 24 remaining men (median=1,119 s), suggesting that sex cannot explain the significantly lower tolerance to treadmill exercise in the diabetic and heavy non-diabetic groups in the present study.

In conclusion, the findings of this preliminary study show that type 2 diabetes mellitus accentuates the exercise intolerance in patients with PAD and intermittent claudication. This effect appears to be mainly a function of greater body weight and/or BMI, rather than of diabetes as such. The fact that the $V \mathrm{O}_{2}$ time constant was significantly related to exercise tolerance and not correlated with BMI raises the question of what, if any, contribution slowed $V \mathrm{O}_{2}$ kinetics makes to exercise intolerance in PAD. This aspect deserves further investigation.

Acknowledgement This study was funded by the National Heart Foundation (Australia). The authors thank B. Sanderson for his technical assistance.

Duality of interest There are no conflicts of interest that might bias this work.

\section{References}

1. Askew CD, Green S, Walker PJ et al (2005) Skeletal muscle phenotype is associated with exercise intolerance in patients with peripheral arterial disease. J Vasc Surg 41:802-807

2. Barker G, Green S, Green A, Walker PM (2004) Walking performance, $\mathrm{VO}_{2}$ kinetics and resting skeletal muscle pyruvate dehydrogenase complex activity in peripheral arterial disease. Clin Sci 106:241-249

3. Hou X-Y, Green S, Askew C, Barker G, Green A, Walker PW (2002) Skeletal muscle mitochondrial ATP production rate and walking performance in peripheral arterial disease. Clin Physiol Funct Imaging 22:81-91

4. Regensteiner JG, Wolfel EE, Brass EP et al (1993) Chronic changes in skeletal muscle histology and function in peripheral arterial disease. Circulation 87:413-421

5. Askew C, Green S, Walker PM (2002) Physiological and symptomatic responses to walking and cycling in peripheral arterial disease. Clin Physiol Funct Imaging 22:348-355

6. Sorlie D, Myhre K (1978) Lower leg blood flow in intermittent claudication. Scand J Clin Lab Invest Suppl 38:171-179

7. Green S, Askew C (2001) The physiology of walking performance in peripheral arterial disease. In: Maffuli N, Chan KM, MacDonald R, Malina RM and Parker AW (eds) Sports medicine for specific ages and abilities. Churchill-Livingstone, Edinburgh, pp 385-393

8. Hiatt WR (2001) Medical treatment of peripheral arterial disease and claudication. N Engl J Med 344:1608-1621

9. Diehm C, Schuster A, Allenberg JR et al (2004) High prevalence of peripheral arterial disease and comorbidity in 6880 primary care patients: cross-sectional study. Atherosclerosis 172:95105

10. Hiatt WR, Hoag S, Hamman RF (1995) Effect of diagnostic criteria on the prevalence of peripheral arterial disease: the San Luis Valley Diabetes Study. Circulation 91:1472-1479

11. Murabito JM, D'Agostino RB, Silbershatz H, Wilson WF (1997) Intermittent claudication: a risk profile from the Framingham Heart Study. Circulation 96:44-49

12. Hughson WG, Mann JI, Garrod A (1978) Intermittent claudication: prevalence and risk factors. Br Med J 1:1379-1381

13. Reunanen A, Takkunen H, Aromaa A (1982) Prevalence of intermittent claudication and its effect on mortality. Acta Med Scand 211:249-256

14. Stoffers HEJH, Kester AM, Kaiser V, Rinkens PELM, Knottnerus JA (1997) Diagnostic value of signs and symptoms associated with peripheral arterial occlusive disease seen in general practice: a multivariable approach. Med Decis Mak 17:61-70

15. Regensteiner JG, Sippel JM, McFarling ET, Wolfel EE, Hiatt WR (1995) Effects of non-insulin dependent diabetes on maximal exercise performance. Med Sci Sports Exerc 27:875-881

16. Regensteiner JG, Bauer TA, Reusch JEB et al (1998) Abnormal oxygen uptake kinetic responses in women with type II diabetes mellitus. J Appl Physiol 85:310-317

17. Wei M, Gibbons LW, Mitchell TL, Kampert JB, Lee CD, Blair SN (1999) The association between cardiorespiratory fitness and impaired fasting glucose and type 2 diabetes mellitus in men. Ann Intern Med 130:89-96

18. Oka RK, Sanders MG (2005) The impact of type 2 diabetes and peripheral arterial disease on quality of life. J Vasc Nurs 23:61-66

19. Katzel LI, Sorkin JD, Powell CC, Gardner AW (2001) Comorbities and exercise capacity in older patients with intermittent claudication. Vasc Med 6:157-162

20. Dolan NC, Liu K, Criqui MH et al (2002) Peripheral artery disease, diabetes, and reduced lower extremity functioning. Diabetes Care 25:113-120

21. Sanderson B, Askew C, Stewart I, Gibbs H, Walker PJ, Green S (2006) Short-term effects of cycle and treadmill training on exercise tolerance in peripheral arterial disease. J Vasc Surg 44:119-127

22. Labs KH, Dormandy JA, Jaeger KA, Stuerzebecher CS, Hiatt WR (1999) Transatlantic conference on clinical trial guidelines in peripheral arterial disease: clinical trial methodology. Circulation 100:e75-e81

23. Barker G, Green S, Walker PM (2004) Effect of carbohydrate supplementation on walking performance in peripheral arterial disease: a preliminary physiologic study. J Vasc Surg 40:932938

24. Bauer TA, Regensteiner JG, Brass EP, Hiatt WR (1999) Oxygen uptake kinetics during exercise are slowed in patients with peripheral arterial disease. J Appl Physiol 87:809-816

25. Angelides N, Nicolaides A, Needham T, Dudley H (1978) The mechanism of calf claudication: studies of simultaneous clearance of ${ }^{99} \mathrm{Tc}^{\mathrm{m}}$ from the calf and thigh. Br J Surg 65:204-209

26. Green S (2002) Haemodynamic limitations and exercise performance in peripheral arterial disease. Clin Physiol Funct Imaging 22:81-91

27. van der Feen C, Neijens FS, Kanters SD, Mali WP, Stolk RP, Banga JD (2002) Angiographic distribution of lower extremity atherosclerosis in patients with and without diabetes. Diabet Med 19:366-370 